\title{
An Analyzable Annotation Language
}

\author{
Sarfraz Khurshid Darko Marinov Daniel Jackson \\ Laboratory for Computer Science \\ Massachusetts Institute of Technology \\ 200 Technology Square, Cambridge, MA 02139 \\ $\{$ khurshid,marinov,dnj\}@lcs.mit.edu
}

\begin{abstract}
The Alloy Annotation Language (AAL) is a language (under development) for annotating Java code based on the Alloy modeling language. It offers a syntax similar to the Java Modeling Language (JML), and the same opportunities for generation of run-time assertions. In addition, however, AAL offers the possibility of fully automatic compile-time analysis. Several kinds of analysis are supported, including: checking the code of a method against its specification; checking that the specification of a method in a subclass is compatible with the specification in the superclass; and checking properties relating method calls on different objects, such as that the equals methods of a class (and its overridings) induce an equivalence. Using partial models in place of code, it is also possible to analyze object-oriented designs in the abstract: investigating, for example, a view relationship amongst objects.
\end{abstract}

The paper gives examples of annotations and such analyses. It presents (informally) a systematic translation of annotations into Alloy, a simple first-order logic with relational operators. By doing so, it makes Alloy's automatic analysis, which is based on state-of-the-art SAT solvers, applicable to the analysis of object-oriented programs, and demonstrates the power of a simple logic as the basis for an annotation language.

\section{Categories and Subject Descriptors}

D.1.5 [Programming Techniques]: Object-oriented Programming; D.2.1 [Software Engineering]: Specifications; D.2.4 [Software Engineering]: Program Verification-Class invariants, programming by contract, formal methods.

\section{General Terms}

Verification, Languages

\section{Keywords}

Specification language, Java language, Alloy modeling language, Alloy Analyzer, compile-time analysis

Permission to make digital or hard copies of all or part of this work for personal or classroom use is granted without fee provided that copies are not made or distributed for profit or commercial advantage and that copies bear this notice and the full citation on the first page. To copy otherwise, to republish, to post on servers or to redistribute to lists, requires prior specific permission and/or a fee.

OOPSLA'02, November 4-8, 2002, Seattle, Washington, USA.

Copyright 2002 ACM 1-58113-417-1/02/0011 ...\$5.00

\section{Introduction}

The benefits of annotating code with specifications are well known, but for the most part they have not been realized, and programmers still view them as more trouble than they are worth. Much progress has been made. Annotation languages are now better integrated with programming languages; they can handle the complexities of object-oriented code (such as dynamic dispatch, and the interaction between frame conditions and subclassing); and they can be readily compiled into run-time assertions. But to make annotations attractive to practitioners, we believe it is necessary to squeeze more value from them, by providing new analyses for the same annotations.

In this paper, we outline an annotation language, called AAL, that we are currently developing. The paper illustrates AAL on small examples. We have not yet demonstrated that the features of the language, or its analysis technology, will scale to realistic programs. AAL is similar to the Java Modeling Language (JML) [19] in its aim of providing a lightweight approach to code annotation, and it has adopted the JML syntax for distinguishing pre- and poststate values. JML, however, uses Java expression syntax, and is conceptually founded on algebraic specifications [18]; AAL, in contrast, is based on a simple first-order logic with relational operators, and is thus more in the tradition of semantic data modeling (now called "object modeling").

AAL allows partial specifications of methods to be written, which can be executed as run-time assertions. In addition, however, it supports fully automatic compile-time analysis. This analysis, which is based on our previous work, and reduces to finding models of relational formulas, has a variety of applications. It can be used to check code against specifications, producing counterexample traces that show how a method's code misbehaves. It can be used to generate test cases from invariants and preconditions fully automatically; this is especially useful for elaborate data structures, such as trees, which cannot be generated randomly because of intricate structural constraints, and are tedious to generate manually. It can be used also for more elaborate checks that are easily expressed in our logic but not usually expressible at all in other annotation languages, such as that an equals method actually defines an equivalence.

Automatic compile-time analysis, more than run-time analysis, has the potential to change how programmers work. The success of test-first programming, a key practice of Extreme Programming [1], indicates not only the importance of hav- 
ing regressions tests (and having them early), but also the value of focusing on intent before coding. Even though the programmer isn't required to write a specification, the act of devising test cases forces a consideration of what behavior is intended. Our approach promises to bring the benefit of test-first programming with lower cost. An invariant (especially for a complex structure) is usually much less work to write than a reasonable test suite. Having written it, the programmer can rely on our analysis not only to generate a high quality test suite but also to check crucial properties of the specification itself. A crucial advantage of compile-time analysis is that it can be applied before the code is complete, whereas run-time analyses require not only that the module be complete, but that whatever libraries it uses be complete and available also.

The language we present is a declarative, first-order logic. This makes it simple and familiar. The inclusion of relational operators makes it easy to express navigations amongst objects. The set of elements of a list, for example, may be denoted by the expression p.*next.elt, where $p$ is the variable that points to the list, *next indicates zero or more traversals of a field next from list nodes to list nodes, and elt indicates traversal of a field from a node to its contained element. The user can view the heap as a labeled graph, modeled with standard notions of sets and relations. We believe this is simpler than an algebraic viewpoint, which requires a collection of predefined datatypes to model sets, tuples, sequences, and so on.

The logic is expressive enough to capture much of the behavior of an object-oriented program. In particular, it can express the mutation of shared objects that is the cause of much complexity in object-oriented programs. The annotation language is actually a full modeling language, and can be used to model and analyze an object-oriented program in its entirety. We illustrate this by application to the design of views, in which mutation of one object is propagated to another belonging to a different class [7].

Previous work has shown how to use the Alloy language to represent the heap of an object-oriented program [12,13], how to check code automatically against an Alloy specification [15], and how to generate test cases automatically from Alloy invariants [24]. The new contributions of this paper are:

- An embedding of the Alloy logic in an annotation language designed to be as close as possible to JML;

- A translation scheme that translates annotations and checks into simple Alloy formulas, making existing Alloy analysis technology [9] applicable;

- As part of this scheme, a handling of some of the tricky aspects of object-oriented programs and specifications, most notably: frame conditions (and their interaction with object sharing and subclassing), dynamic dispatch, recursion, and user-defined equality;

- A series of small examples illustrating the power of the approach, including analyzing whether a user-defined equality method obeys the object contract, and analyzing views (as used extensively in the Java Collections Framework [34]).

Our approach can be viewed as an attempt to gain the benefits of two complementary approaches to annotation: the JML approach [19], which allows rich specifications but only run-time analysis, and the Extended Static Checker (ESC) approach [4], which provides compile-time analysis but for much less expressive specifications. This work builds on both of these projects. From JML, it takes ideas of syntax and specification inheritance. From ESC, it takes ideas about how to interpret frame conditions. It combines these ideas with an analysis technology that makes it possible to have both rich specifications and compile-time analysis.

\section{Overview}

The key idea in this paper is that the behavior of an objectoriented program can be modeled and analyzed within a simple first-order logic of sets and relations. In this section, we explain briefly how the state of an object-oriented program is modeled with sets and relations; how a method's behavior is modeled as a formula; and how the problem of analysis always reduces to finding an assignment of (set and relation) values to variables that makes a formula true. In later sections, these ideas are elaborated more fully.

\subsection{Modeling state}

The heap of an executing program is viewed as a labeled graph whose nodes represent objects and whose edges represent fields. The presence of an edge labeled $f$ from node $o$ to $o$ ' says that the $f$ field of the object $o$ points to the object $o$ '. Mathematically, we treat this graph as a set (the set of nodes) and a collection of relations, one for each field. From a type perspective, the relations are untyped, and so any object may be associated by a given field with any other. We therefore classify the objects into classes by associating a subset with each class, and we constrain the relation associated with a field so that it only maps objects that belong to the field's class.

This simple approach, in which fields are essentially untyped, has a great advantage. It eliminates the need for casts. The assertion that an object belongs to a given class becomes a membership test. And since field "get" (dereferencing) is treated as relational image, application of a field to an object of the wrong class yields the empty set. This is particularly handy in navigation expressions, in which a field is applied over a set of objects, only some of which belong to the appropriate class.

To model mutation, we simply associate a distinct graph with each state. In a specification there are only two statesthe pre-state and the post-state. Mathematically, we treat fields as ternary relations, each of which maps an object to an object in a given state.

\subsection{Specifications and models}

Our annotation language includes the following parts. Each method may be annotated with three formulas - a precondition (labeled requires), a postcondition (labeled ensures), 
and a behavior model (labeled does) - and a frame condition (labeled modifies). The precondition, postcondition and frame condition together form a specification, which is used to check the code of the method. In the checking of a client, the specification may be used as a surrogate. Sometimes, however, a client may legitimately rely on stronger properties than the specification guarantees, because, for example, the specification has been deliberately weakened to ensure that subclasses are subtypes. Or we may want to perform an analysis of the method that accounts for the details of its behavior. In these cases, it is more appropriate to use the behavior model than the specification. This model may be provided explicitly by the user (especially if no code is yet present), but more commonly it will have been automatically generated from the code, or from the specification (by conjoining pre- and post-conditions). We thus use the terms specification and (behavior) model interchangeably, except when we want to point out the distinction.

\subsection{Dynamic dispatch}

The specification or behavior model of a method may "invoke" other methods as if they were mathematical functions. This is necessary for specifying polymorphic methods. A method to insert an element into a set, for example, will use the equals method of the element to determine whether or not the element should be inserted. To express this in the specification or model, we use equals as if it were a mathematical function. In order to capture the dynamic dispatch in which the meaning of this function depends on the class the element belongs to, this function is desugared to a wrapper function that invokes the equals function of the appropriate class based on which class the element belongs to.

\subsection{Analysis}

Our analysis works by translating theorems to be checked into Alloy [10,14], a software modeling language. We use Alloy's analysis tool, the Alloy Analyzer, to check the theorems. The analysis always reduces to finding an assignment for a formula that binds its free variables to values in such a way that the formula is true. In general, the free variables will represent the state of the program at several points in its execution. To check that a method in a subclass correctly preserves the specification of its superclass method, for example, we would construct a formula saying that a pre- and post-state exist that satisfy the constraints of the behavioral model of the subclass method, but do not satisfy the constraints of the superclass specification. A solution to this formula is a pair of states illustrating a violation: that is, a counterexample.

Our language is undecidable, so the analysis cannot be complete. The Alloy Analyzer conducts a search in a "scope" specified by the user that bounds the size of the basic types. In application to program checking, this amounts to limiting the size of the heap. A failure to find a counterexample does not mean that the theorem holds; it may mean that a counterexample exists, but only for heaps larger than those considered. In our experience in analyzing design models, we have found that surprisingly small scopes suffice to detect many subtle errors, and it seems likely that the same should hold for code.
The reader may wonder how this approach differs from testing; after all, only some finite set of cases is considered. There are two crucial differences. First, the user does not have to provide any test cases. Second, experience with model checking in the last decade has demonstrated that exhaustive analysis of a bounded space is far more effective at finding bugs than testing, largely because of the large number of cases considered. The satisfiability solvers that we use as the underlying technology in the Alloy Analyzer can routinely handle spaces of several hundred bits in width; an analysis of a billion cases (or, a state space of 30 bits), which is completely infeasible in testing, is thus very small.

\section{Examples}

Our presentation is organized around two examples. Both illustrate how programs that are seemingly straightforward can become surprisingly complicated. In the first, we consider determining whether the equals method of a class satisfies the "object contract" - in particular that it define an equivalence relation over the elements of the class. In practice, it is easy to get this wrong, especially when overriding the method in a subclass, because of the asymmetry of method dispatch.

In the second, we consider two implementations of a set interface: one a standard implementation as a red-black tree, and the other as a view on the first. This second class exhibits strange properties. Modifying the subset class causes modifications in the other; worse, because a subset is defined with a predicate on elements, inserting an element that does not satisfy the predicate will not cause it to be inserted at all. A set object thus fails to obey the simplest set axioms: that inserting an element into a set results in it belonging to the set afterwards. In fact, even the complexities of views are not necessary to break these axioms; since an inserted object may replace an object already present that is equivalent (by its equals method), insertion may actually remove an object! These are not contrived examples. It is for reasons such as these that the specification of the set interface in the Java Collections Framework does not claim that the standard set axioms hold.

\subsection{Overriding equals}

We first use AAL to specify the behavior of two Java equals methods and to check the correctness of their overriding. Any implementation of equals should satisfy two crucial properties: at least being an equivalence relation and being consistent with hashCode [34]. It is easy to develop such implementations incorrectly [30], and special care should be taken for classes that allow equals to be overridden, i.e., for classes that are not final.

\subsubsection{Superclass}

Consider the equals method of the class java.awt.Dimension that is a non-final class from the standard Java libraries [34]. An object of java.awt.Dimension has two integer fields, width and height. The following is an example AAL annotation of java.awt.Dimension and its equals method: 


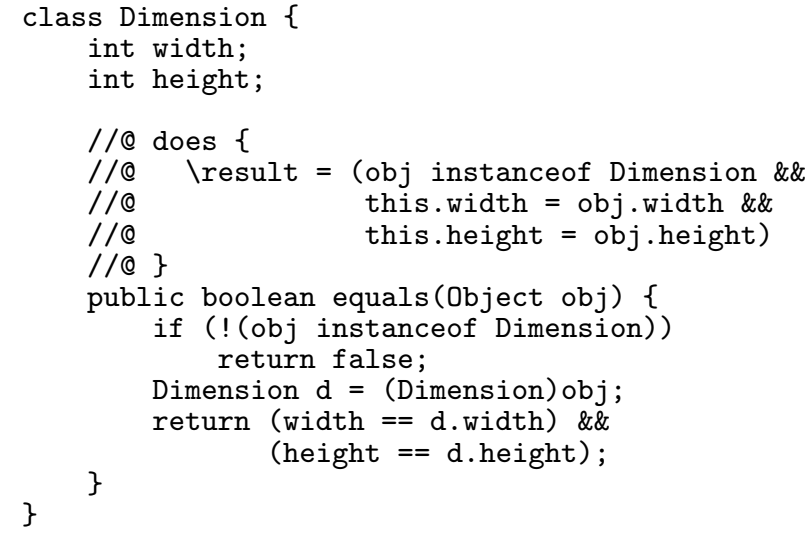

The does annotation specifies a model for the behavior of the method. Such models can be provided by the programmer or automatically generated from the code, as here. A model is a formula over the method parameters, classes, and fields. The keyword $\backslash$ result, borrowed from JML, allows referring to the return value of the method. In this example, we consider an annotation that provides a model and not a specification, because we want to check that the model correctly implements the specification.

\subsubsection{Checking AAL models}

Every AAL specification and model can be automatically translated into Alloy and checked with the Alloy Analyzer (AA). ${ }^{1}$ We describe in this section only the result of the analysis; Section 5 presents the translation. We use AA to automatically check that all equals methods implement an equivalence relation. The following AAL assertion expresses the equivalence property:

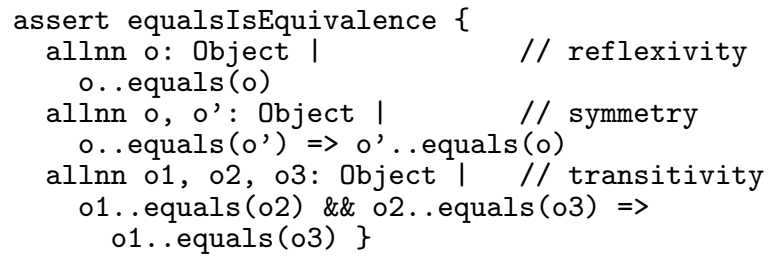

This assertion consists of three, implicitly conjoined, formulas. The variables quantified with allnn range over all nonnull objects. The operator ' $\Rightarrow$ ' denotes implication. The operator '. .' invokes AAL methods, using (single) dynamic dispatch as in Java, based on the dynamic class of the receiver object. Note that this property is inherently global, i.e., an invariant on all classes and not just a class invariant for Object.

The assertion is checked by presenting a translation into basic Alloy (described later in Section 5) to the Alloy Analyzer. The expressions involving equals are expanded using the behavior model of the Dimension class given above. In this case, the analyzer checks the assertion, and reports that there are no counterexamples for this particular class.

\footnotetext{
${ }^{1}$ We have not yet implemented the translation; for the examples of this paper we performed it manually.
}

\subsubsection{Subclassing with overriding}

Consider Dimension3D, a subclass of java.awt.Dimension that adds a field depth and incorrectly overrides equals:

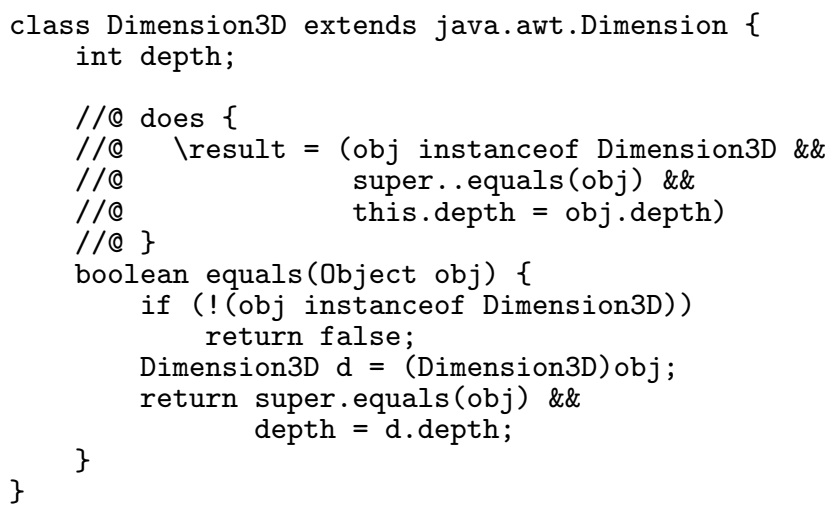

We again use AA to check the assertion equalsIsEquivalence, and this time it automatically generates a counterexample:

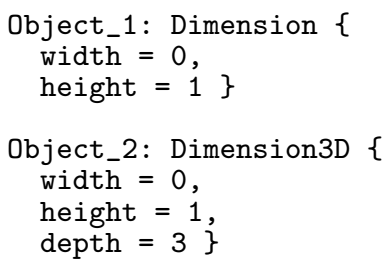

These two objects violate the symmetry property: setting o $=$ Object $\_1$ and $o^{\prime}=$ Object $\_2$ makes o. equals $\left(o^{\prime}\right)$, but not $0^{\prime}$.. .equals (o). The reason is that the former equals is from Dimension, and it is oblivious of the field depth introduced in Dimension3D, whereas the latter equals is from Dimension3D, and it correctly compares all the fields in the objects. This counterexample shows that Dimension3D incorrectly overrides equals, as it violates the equivalence property.

The problem is actually in the equals method of the class java.awt.Dimension; it is hard to correctly override it in a subclass. An overridable equals can be implemented in Java using the getclass method instead of the instanceof primitive [30]. In our running example, it requires changing equals of java.awt.Dimension to use the expression obj.getClass() $==$ this.getClass () instead of obj instanceof Dimension.

Modeling this change in AAL is straightforward; we replace obj instanceof Dimension

with

$$
\text { obj } \cdot \operatorname{getClass}()=\operatorname{get} C l a s s()
$$

in does. We translate the changed AAL specification into Alloy and again use AA to check the equivalence assertion. This time AA reports no counterexamples.

\subsection{Subset views}

We next illustrate AAL by developing specifications for sets and subset views. We consider a generalization of views returned by the subSet method in the class java.util. TreeSet of the Java Collections Framework (JCF). We develop example specifications for parts of the following: 
- The java.util.Set interface.

- The abstract class java.util. AbstractSet for sets whose element membership is based on equals method. This specification is abstract and uses an Alloy set to represent the set of elements.

- The java.util.TreeSet class that uses red-black trees to implement java.util.AbstractSet.

- A set that represents a subset view on another set; this specification also shows how a method argument that is essentially a function can be handled.

Finally, we illustrate how behavior of methods can be automatically analyzed in an interactive fashion.

\subsubsection{Set interface}

Consider the following example AAL specification for a part of the java.util.set interface:

package java.util;

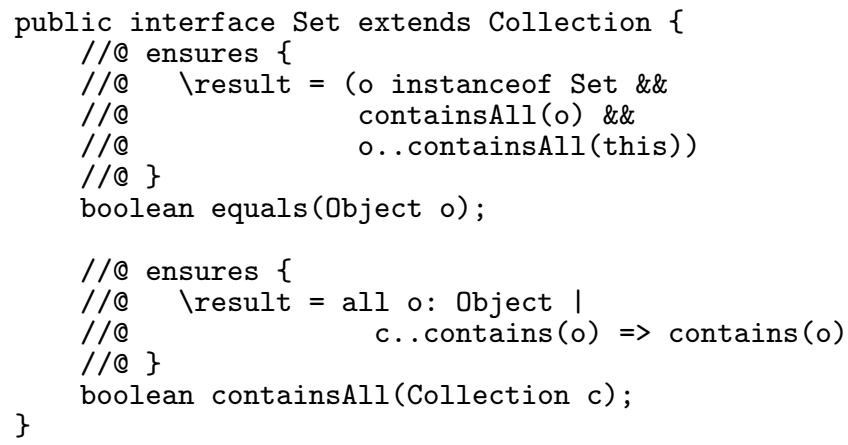

The specifications are given only for methods equals and containsall, because the interface set should not specify all standard axioms for sets. Otherwise, subclasses such as Subset view could not be subtypes of Set, as noted earlier. We present in the example only the most relevant parts of the specification; AAL additionally borrows from JML parts that specify exceptional behaviors (when a method returns throwing an exception), access modifiers (public, protected, and private), inheritance of specifications etc.; for details see [19].

Since the specifications omit preconditions (there are no requires annotations), they are true by default, as in JML. The postcondition for equals specifies that the result is true iff $\circ$ is a non-null object of set or its subclass and this and o are subsets of each other. The method containsAll determines if $c$ is a subset of this; the result is true iff every object contained in $c$ is also contained in this; ' $:$ ' denotes set membership.

\subsubsection{Abstract set}

Consider next the following AAL specification for a part of the abstract class java.util. AbstractSet that implements the set interface:

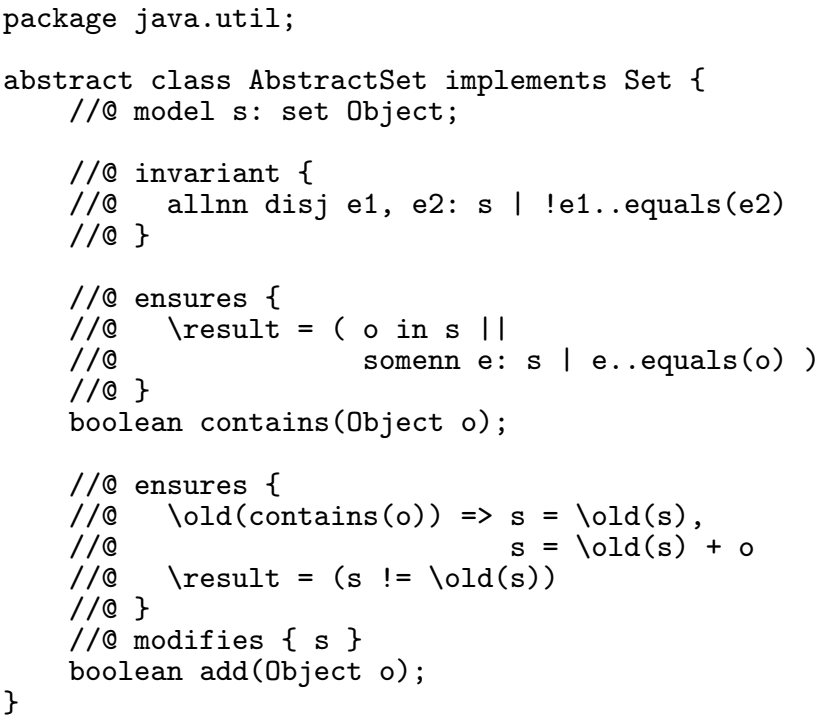

The model field s declares a (mathematical) set of objects; set is an AAL keyword, and ': set' declares a subset. This field is used in specifications, but it does not need to be in an implementation for this class or its subclasses [19]. The class invariant specifies that objects in the set are not equal with respect to the equals method. The keyword disj declares disjoint subsets; in this case, it ensures that e1 and e2 are distinct. The symbol '!' denotes negation.

The postcondition for contains specifies that the result is true iff $\circ$ itself is in $\mathbf{s}$ (which handles the case when $\circ$ is null) or there exists a non-null object in s that is equals of o; 'in' denotes subset/membership operation, and 'II' denotes disjunction.

Each of the methods presented so far is a pure method (also referred to as an observer), i.e., it does not modify the state (there is no modifies annotation) and its specification expresses properties of only one state. The method add is a mutator method that modifies the state and also returns a value. Its specification relates a pre-state (i.e., the state immediately prior to the method invocation) to a post-state (i.e., the state immediately after the method invocation).

In AAL postconditions, as in JML, prefixing an expression with \old indicates its evaluation in the pre-state, e.g., $\operatorname{lold}(\mathrm{s})$ is the value of model field $\mathrm{s}$ in the pre-state, and lold(contains (o)) is an invocation in the pre-state. (It is not legal to apply \old to the invocation of mutator methods.) The formula $F \Rightarrow$ G,H means that if $F$ holds, $G$ should hold also; if not, $\mathrm{H}$ should hold. The postcondition for add specifies that the set is unchanged if it contains the object $\circ$ in the pre-state; otherwise, o is added to the set in the post-state.

The modifies annotation in AAL is the same as in JML: it lists the fields that the method can change; the assignment to other fields is not allowed. We discuss the semantics of modifies in more detail in the next subsection. Intuitively, the method add can change only the field s (of this); all other fields retain their pre-state values. 


\subsubsection{Set implementation}

Consider next the following AAL specification for a part of the class java.util.Treeset that implements sets (of comparable elements) with red-black trees:

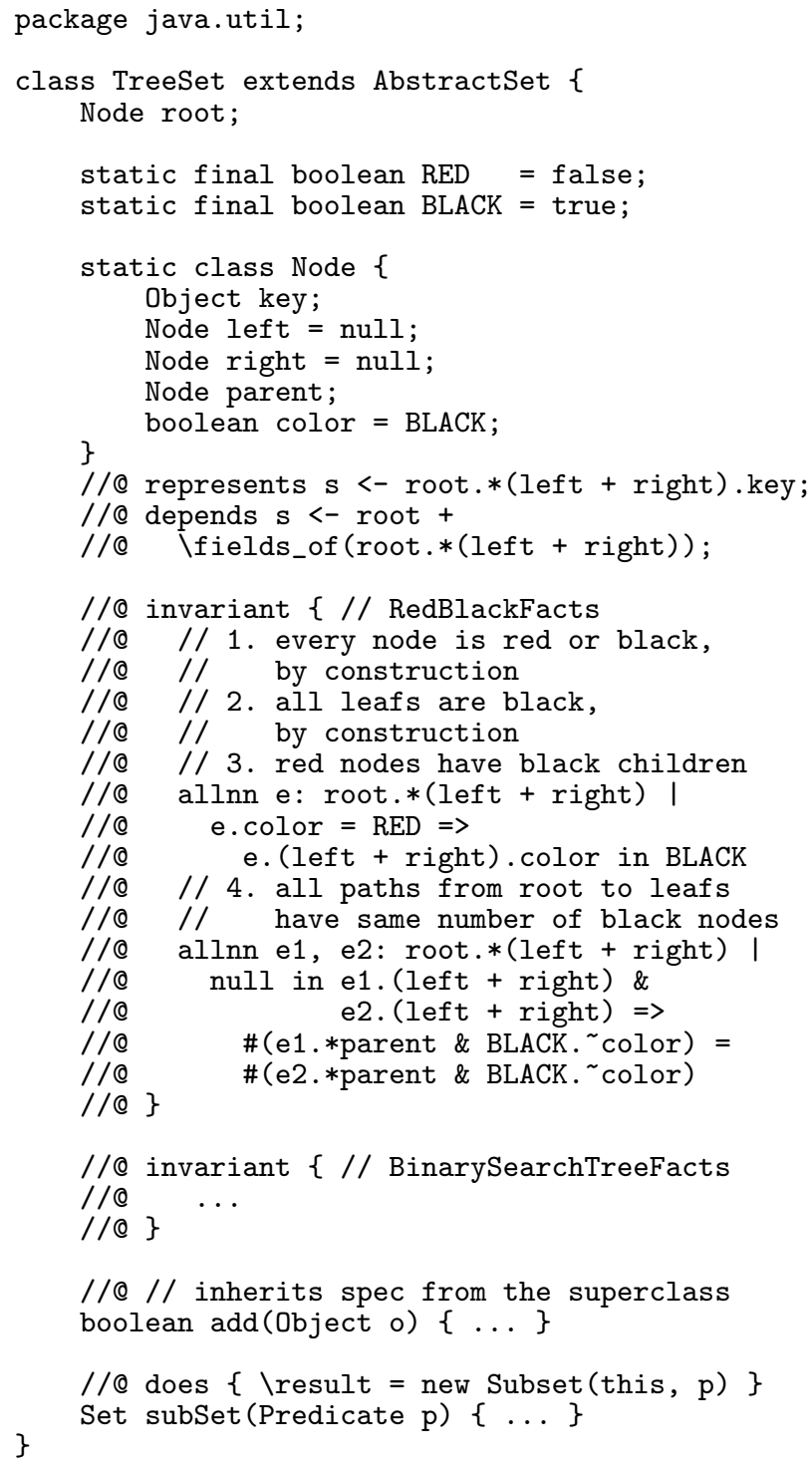

Each tree has a root of class Node; each node has its data stored in key, pointers to its parent and its left and right children, and color which can be RED or BLACK.

The represents annotation specifies the abstraction function, i.e., how the concrete fields in objects that build a TreeSet relate to the model field in Set; '*' denotes reflexive transitive closure, and ' + ' denotes set union. The depends annotation specifies a set of the concrete fields that the model field depends on; \fields_of represents all fields for each object in the given set.

The semantics of modifies takes into account these dependencies: if a method can modify a model field $f$, then it can also modify the fields that $f$ depends on. In the example, the add method in TreeSet can modify all fields in the tree, since add in AbstractSet can modify field s. Conceptually, the depends annotation allows mutator methods in TreeSet to inherit their specifications from AbstractSet without any change, thus enabling modular reasoning. The full treatment of modifies is beyond the scope of this paper; details can be found in $[20,29]$.

The class invariant specifies the structural constraints for a red-black tree. All four properties can be easily expressed in AAL; '\#' denotes the size of the set and ' ' denotes the transpose relation, effectively following the field backwards. This example omits the invariants for a binary search tree, such as acyclicity, ordering of elements, and a definition of the parent relation as the transpose of the union of left and right. These invariants are also straightforward to express in AAL [24].

The main property to check for an implementation of a method is code conformance, i.e., whether the code correctly implements the specification. We call a state valid iff all objects in the state satisfy their respective class invariants. Code conformance requires showing that for all valid prestates that satisfy method precondition, the execution of the method body produces a post-state that is valid and satisfies the postcondition. In Alloy, this is schematically expressed as:

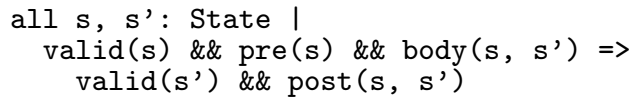

One way to check code conformance is to translate the code of the method body into an Alloy formula and analyze the above implication with the Alloy Analyzer. Jackson and Vaziri developed a technique for automatic translation of a subset of Java into Alloy [15]. Work proceeds on optimizing this approach [36]; although it does not yet scale, it can handle a realistic red-black tree implementation in which the checked procedures have up to 50 lines of code. Another way to check code conformance is to use automatic testing. The TestEra framework [24] generates cases automatically from invariants and has also been demonstrated on the red-black tree implementation.

The method subSet is used to create a subset view. The model for this method uses a constructor for subset that creates a a new view on $\mathrm{s}$ with filter $\mathrm{p}$.

\subsubsection{Subset view}

We next develop an AAL specification of a subset view. In JCF, the class java.util.TreeSet provides an implementation of a (sorted) set, as shown above, and also of a subset view. A subset view is created with so.subSet (fromElement, toElement), where so is the backing set object, and the result is a portion of so with elements ranging from fromElement, inclusive, to toElement, exclusive. The returned set is backed by so, so changes in the view are reflected in so, and viceversa. Note that a view can also be constructed as a view on another subset view.

More generally, a subset view on a set can be created by giving a predicate (boolean returning function) that determines 
membership in the subset. In a language that supports firstclass functions, a view analogous to the above view in JCF could be created by so.subSet(p), where so is a set and p is a predicate defined, for example, as (lambda (o) (and ( $>=0$ fromElement) (< o toElement))) in Scheme. In Java, predicates, and first-class functions in general, are obtained by wrapping them within objects.

The following is a sample AAL annotated abstract class for predicates:

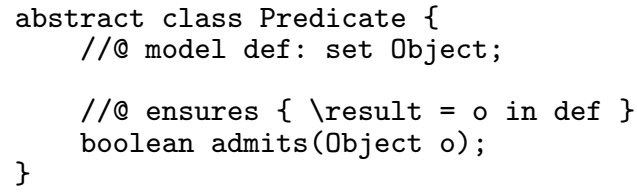

Each Predicate object has a model field def that denotes a (mathematical) set of objects (extensionally) defining the predicate. This field contains the admissible objects, i.e., the objects for which the functional predicate would evaluate to true. The method admits determines if the input object $\circ$ is admissible for this predicate.

The following is an AAL annotated class for subset views:

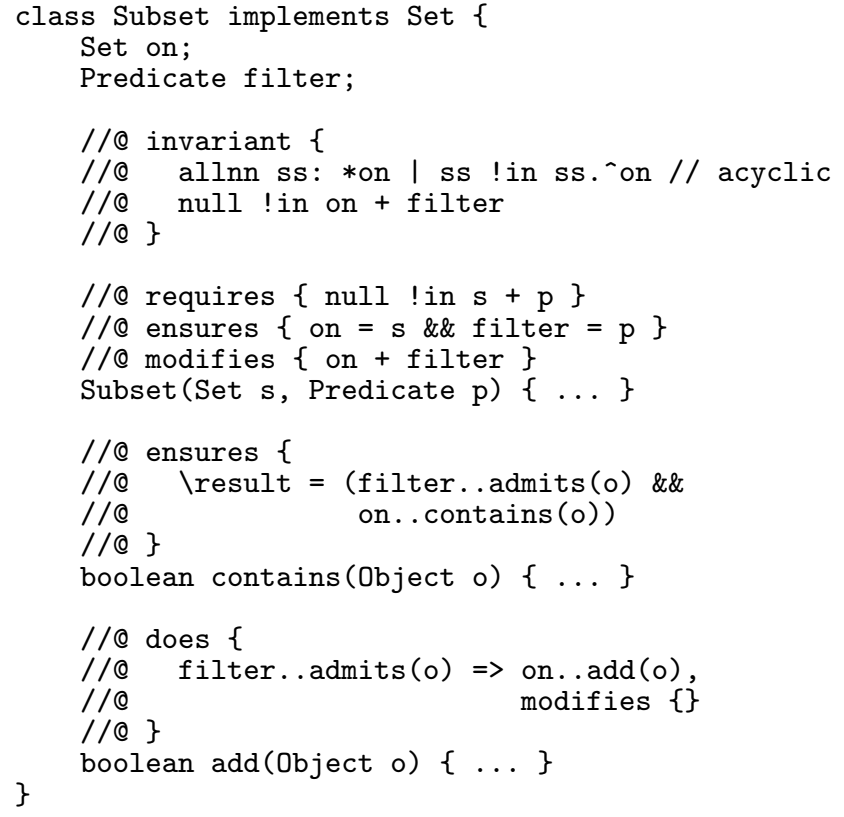

Each Subset object has two fields: on points to the set object that backs this subset view and filter is a predicate that determines membership for this subset view.

The class invariant requires that subset views have no cyclic dependencies along on fields, i.e., that for each subset view, there be (transitively) a backing set that is not a view; the operator ' ' denotes transitive closure.

The constructor creates a Subset from the given set and predicate. The precondition requires that these arguments be non-null. The method contains determines the membership of an object in a subset view. The postcondition requires that the input object $\circ$ be both admissible by the predicate filter and also in the backing set of this. The method add first checks if o is admissible by filter: if so, add simply invokes the method add for the backing set; otherwise, add does not modify any field in the state. Using modifies as a subformula allows the user to specify conditional changes easier than by putting all fields that can change in the modifies clause and listing that they remain the same when some condition holds.

\subsubsection{Analysis}

We next present an example analysis of the above classes. The analysis focuses on a simple property of the behavior of the add methods. We show how to interactively use the analysis results to strengthen some assumptions in the specifications and to recheck the new specifications.

Consider the following annotated code for checking addition:

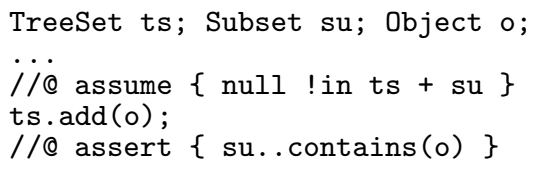

The assume annotation specifies the properties that the code can expect to hold: ts and su are non-null objects. The assert annotation specifies the properties that the code is expected to establish at a certain control point. The above code sequence has only one (mutator) method invocation, and thus only two states. It requires that an addition of an object o to a set ts also make o a member of a subset su. Additionally, the method can assume that the pre-state is valid (all objects in the pre-state satisfy their class invariants), and the method body has to ensure that the post-state is also valid.

The Alloy Analyzer checks (the translation into Alloy of) the above sequence and produces a counterexample, as expected, because su is not constrained to be a subset view on ts. We next add this constraint to our property:

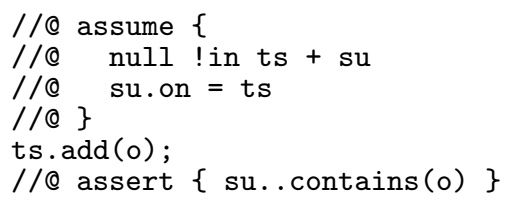

The Alloy Analyzer checks the new sequence and once again produces a counterexample. This time, the assertion fails because the predicate of su does not admit o. We next add a constraint to rule this case out, too:

//@ assume \{

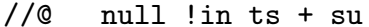

$1 / 0$ su.on $=$ ts

//@ su.filter..admits (o)

//@ \}

ts . add (o) ;

//@ assert $\{$ su..contains(o) $\}$

The Alloy Analyzer checks the new sequence and once again produces a counterexample. This time, the assertion is not 
established because ts contains itself as an element. In this case, invoking ts..contains (ts) (i.e., ts..equals (ts)) is the culprit. In Java, it results in an infinite recursion; in AAL, it results in an underspecified relation as we explain in Section 5.4. We rule out such instances by modifying the class invariant for AbstractSet so that a set does not contain itself (transitively through other AbstractSets):

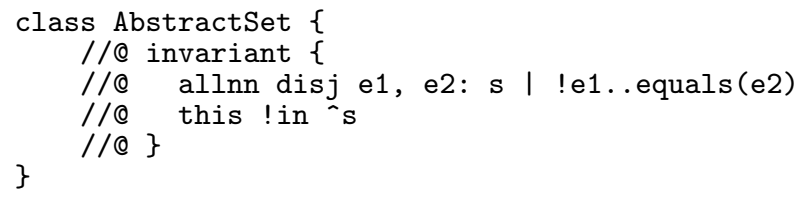

The Alloy Analyzer again checks the sequence and once again produces a counterexample. This time, the assertion fails because ts contains as an element a subset view on ts, i.e., ts indirectly contains itself, which leads to the above problem. One way to rule out these instances is to constrain abstract sets not to include sets as members. (The keyword no requires the set to be empty; ' $\&$ ' denotes set intersection.)

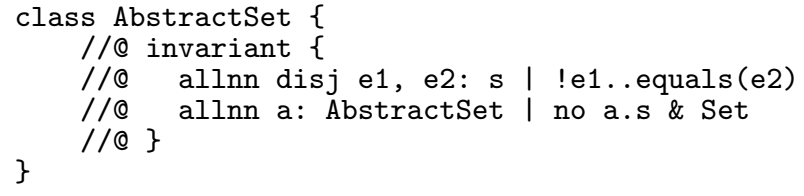

With this correction in place, the Alloy Analyzer checks the sequence and this time reports no counterexamples.

\section{Alloy}

In this section we describe the basics of Alloy; details can be found in $[10,11]$. Alloy is a strongly typed language that assumes a universe of atoms partitioned into subsets, each of which is associated with a basic type. An Alloy model is a sequence of paragraph s that can be of two kinds: signatures, used for construction of new types, and a variety of formula paragraphs, used to record constraints.

\subsection{Signature paragraphs}

A signature paragraph introduces a basic type and a collection of relations (that are called fields) in it along with the types of the fields and constraints on their values. For example,

$$
\text { sig Object }\{\}
$$

introduces Object as an uninterpreted type (or a set of indivisible atoms). A signature may inherit fields and constraints from another signature. For example,

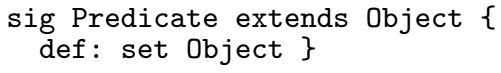

declares Predicate to be a subset of object. The field def declares a relation from Predicate to object. In a field declaration, the keyword set specifies that def maps each atom $\mathrm{p}$ in Predicate to a set of atoms in Object, i.e., $\mathrm{p}$ is an arbitrary relation; the keyword option specifies a partial function; omitting any keyword specifies a (total) function.
In a signature declaration, the keyword static specifies the declared signature to contain exactly one atom. For example, the following declares null to be a singleton subset of Object; a singleton set can be viewed as a scalar:

$$
\text { static sig null extends Object }\{\}
$$

\subsection{Formula paragraphs}

Properties of signature atoms are recorded as logical formulas declared using formula paragraphs. To indicate that such a property always holds, the formula is packaged as a fact paragraph. A property may be defined without imposing it as a permanent constraint by packaging it as a function paragraph to be applied elsewhere. A theorem about a specification or a property that is intended to hold is packaged as an assertion paragraph; a tool can check an assertion by searching for a counterexample, i.e., a model of the formula's negation.

\subsubsection{Relational expressions}

A formula paragraph is formed using Alloy expressions. The value of any expression in Alloy is always a relation - that is a collection of tuples. Each element of such a tuple is atomic and belongs to some basic type. A relation may have any arity greater than one. Relations are typed. Sets are viewed as unary relations.

Relations can be combined with a variety of operators to form expressions. The standard set operators-union $(+)$, intersection (\&), and difference (-) - combine two relations of the same type, viewed as sets of tuples. The dot operator is relational composition. When $\mathrm{p}$ is a unary relation (i.e., a set) and $q$ is a binary relation, p.q is standard composition; $p . q$ can alternatively be written as $q[p]$, but with lower precedence. The unary operators (transpose), - (transitive closure), and * (reflexive transitive closure) have their standard interpretation and can only be applied to binary relations.

\subsubsection{Formulas and declarations}

Expression quantifiers turn an expression into a formula. The formula no e is true when e denotes a relation containing no tuples. Similarly, some e, sole e, and one e are true when e has some, at most one, and exactly one tuple respectively. Formulas can also be made with relational comparison operators: subset (written : or in), equality $(=)$ and their negations (!:, ! in, !=). So e1:e2 is true when every tuple in (the relation denoted by the expression) e 1 is also a tuple of e2. Alloy provides the standard logical operators: \&\& (conjunction), II (disjunction), $\Rightarrow$ (implication), and ! (negation); a sequence of formulas within curly braces is implicitly conjoined.

A declaration is a formula $\mathrm{v}$ op e consisting of a variable $\mathrm{v}$, a comparison operator op, and an arbitrary expression e. Quantified formulas consist of a quantifier, a commaseparated list of declarations, and a formula. In addition to the universal and existential quantifiers all and some, there is sole (at most one) and one (exactly one). In a declaration, part specifies partition and disj specifies disjointness; they have their usual meaning. 


\subsubsection{Functions, facts and assertions}

A function (fun) is a parametrized formula that can be applied by binding its parameters to expressions whose types match the declared parameter types. By default, a function returns a boolean value - the value of the formula in its body. A function may return a (non-boolean) relational value.

Functions can be grouped around the type of the first argument, like the methods of a class in an object-oriented language. For example, a "receiver" and a "result" argument can be declared with the following shorthand:

fun $S:: f(\ldots): T\{\ldots\}$

This declaration is equivalent to

fun $f($ this: $S$, result: $T, \ldots)\{\ldots\}$

where this and result are (reserved) keywords that name the arguments declared anonymously, and the ellipsis in the new argument list is the old argument list. Note Alloy's convention of treating the second argument in a function declaration as the function's result.

A function can also be applied with its first argument presented in prefix position; for example,

$$
\text { s..f }(a, b, c)
$$

is short for

$$
f(s, a, b, c)
$$

A fact is a formula that takes no arguments and need not be invoked explicitly; it is always true. An assertion (assert) is a formula whose correctness needs to be checked, assuming the facts in the model.

\section{Translation}

In this section, we describe the translation of AAL annotations and Java code into Alloy. We organize the presentation of our translation by the constructs that AAL supports. We illustrate each construct using the views example from Section 3 .

\subsection{Inheritance}

The translation of inheritance into Alloy is similar to compilation of OO languages, involving creation of virtual function tables. Details can be found in [25]. In outline, the translation has six steps:

1. Compute a hierarchy of class declarations.

2. Construct sig object that represents the set of all objects in the heap.

3. Translate each class declaration into a sig declaration, with appropriate subset relationship.

4. Translate each method specification into an Alloy function with a unique name.

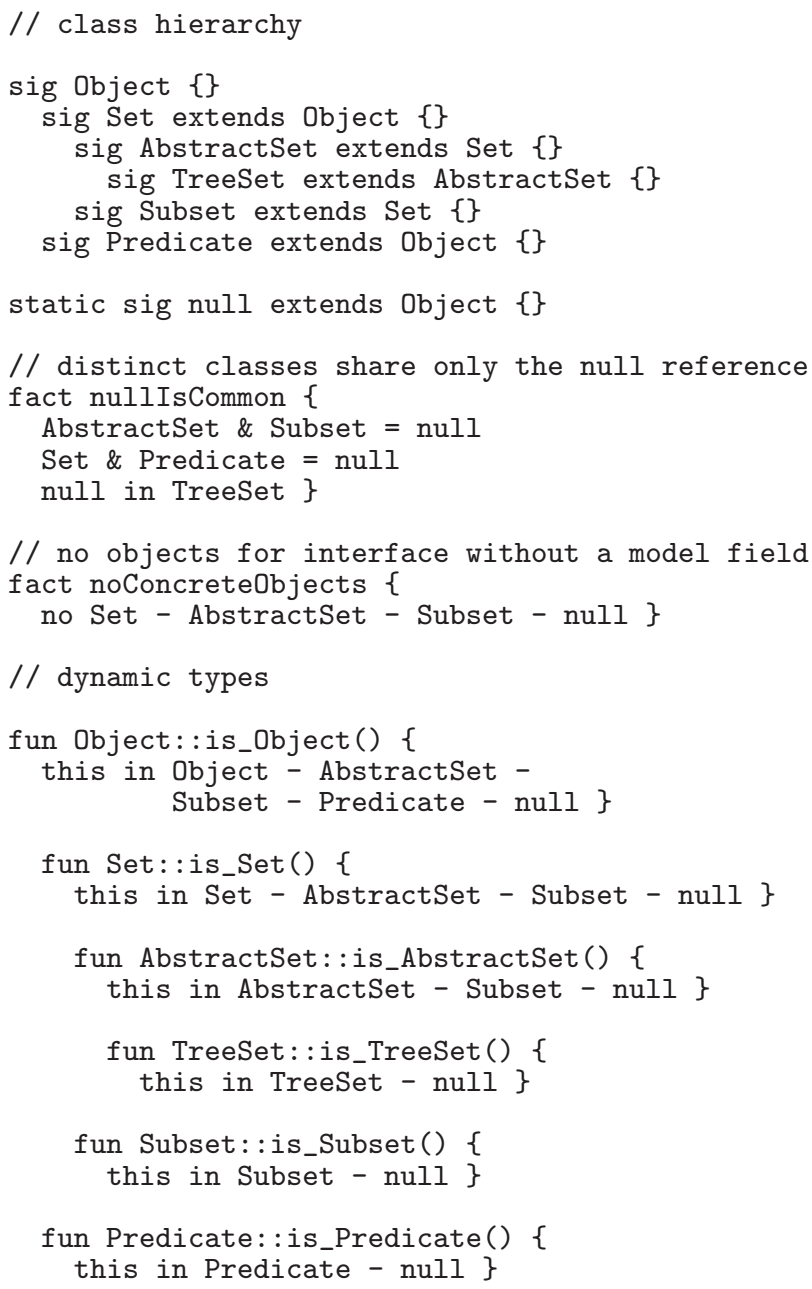

Figure 1: Translation of the class hierarchy for the subset view example.

5. Add dispatching functions that model dynamic dispatch based on the receiver type.

6. Replace super with appropriate static invocation.

We illustrate these steps by translating the equals methods for sets presented in Section 3. Throughout, the Alloy text that results has been indented to show its correspondence to the class hierarchy.

Step 1 computes the following class hierarchy for our running example:

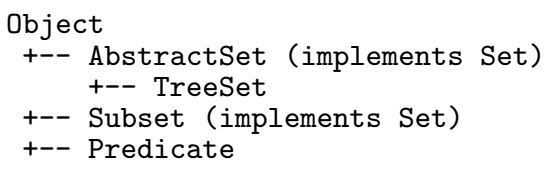

Figure 1 shows the translation done by steps 2 and 3 . The translation introduces null as an atom of object that is shared by all classes. We have previously modeled null as the empty set, but that approach is too restrictive; for example, it disallows null to be in a set of objects. The current 
approach uses the following semantics for null: dereferencing null along a field produces the empty set and invoking a method on null always results in an unsatisfiable formula, as we show in the translation of methods.

Step 4 introduces Alloy functions for the method specifications and behaviors. Since Alloy does not have dynamic dispatch, each function has to have a unique name. For example, the translation for the equals methods introduces the following functions:

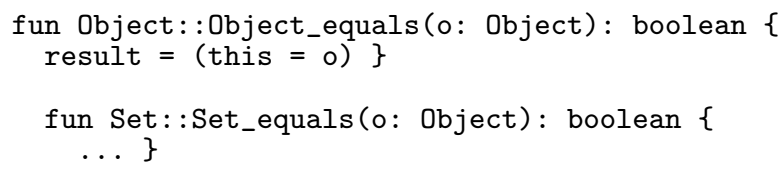

The function corresponding to the (default) equals method in class object is built from its behavior. We show later how the translation builds the body for the function that corresponds to the specification for the Set interface.

Step 5 adds the dispatching function for equals:

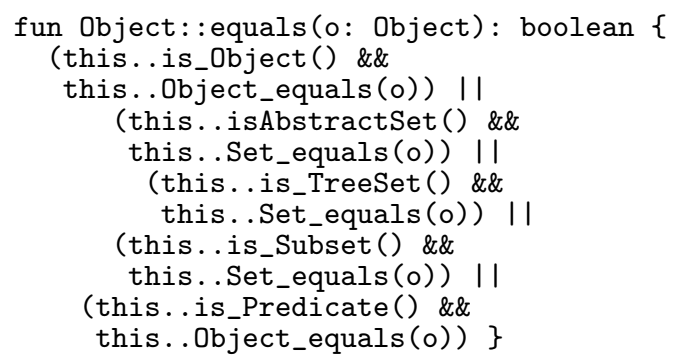

This function models dynamic dispatch based on the receiver type. It allows method invocations o..equals $(p)$ from AAL to be translated into identical function applications, o.. equals ( $p$ ), in Alloy. (Recall that '..' in an Alloy expression denotes function application without dynamic dispatch semantics.) Since Predicate does not override equals, it inherits equals from Object; likewise, AbstractSet, TreeSet, and Subset inherit equals from set.

Step 6 uses the class hierarchy information to replace invocations on super with appropriate static invocations; this step does not translate anything in our running example.

\section{$5.2 \quad$ State}

To handle mutator methods and their sequencing, the translation introduces a model of state in Alloy. We adopt a relational model of the state/heap where fields of objects are treated as relations among objects. A valuation of these relations defines a state; different valuations give rise to different states. The translation first introduces a new signature State and then translates methods that express properties on state(s). For our running example, the signature State is:

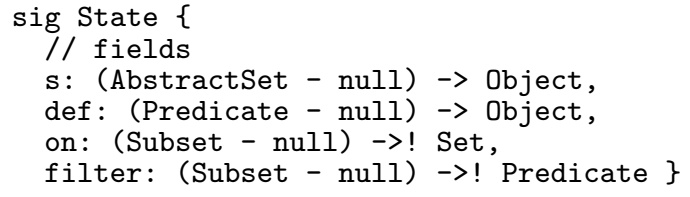

Each atom of State models a state. Each relation in State corresponds to one of the field declarations in the AAL specifications of the classes. (In practice, the names of the fields in State are fully qualified with their class names.) This translation essentially flattens out the hierarchical structure of the heap. The multiplicity marking '!' on on and filter specifies that for each atom $t$ of State, $t . o n$, respectively t.filter, is a total function from non-null subset atoms to Set atoms.

\subsection{Methods and class invariants}

AAL annotations of methods and class invariants are translated into Alloy functions. We first illustrate translation of the class invariant for the Subset class:

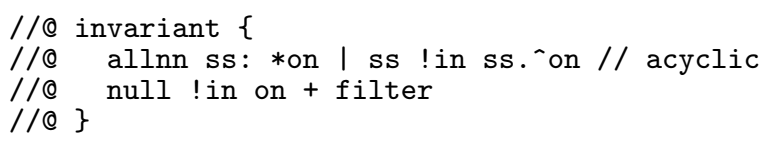

The translation generates the following Alloy function:

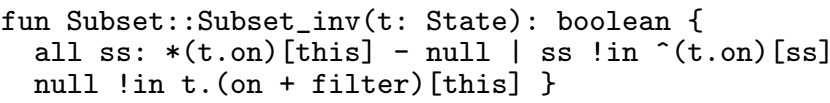

The function name is chosen according to the rules for handling inheritance, and the function has one parameter of type State. Each field access in the body, in this example this. (on + filter), is replaced with the access of the corresponding value in the state, i.e., t. (on + filter)[this]. JML allows class invariants to be inherited, and thus a body for a class invariant may be, in general, conjoined with the class invariants from the superclass(es).

The translation also introduces an Alloy function that determines whether a given state is valid, i.e., whether all objects satisfy their class invariants:

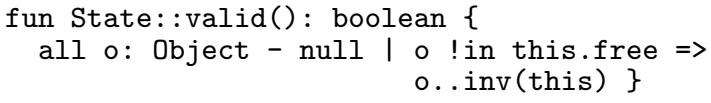

(The free set of unallocated objects is introduced in Section 5.5.) The function inv is the dispatcher for class invariants. Like the equals function shown above, it wraps the functions of the individual classes, dispatching by testing membership of the receiver object in the sets that correspond to the classes.

We next illustrate translation of a pure method, using the following example from AbstractSet:

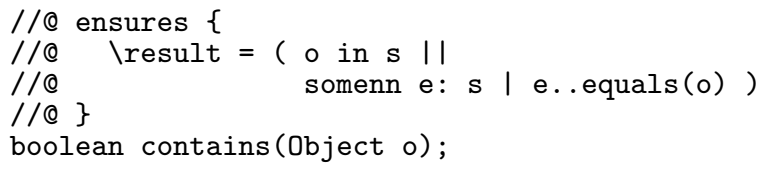

In this example, the method is annotated with a postcondition and the translation uses it to model the method. When a method has a does annotation, the translation can use it for the model.

The translation generates the following Alloy function: 


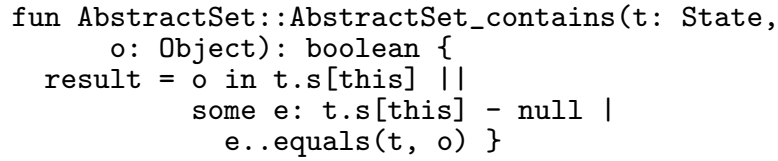

This function has, in addition to the method parameter, one State parameter. This parameter is also added to each invocation in the method body; each of these invocations is on a pure method, since pure methods cannot invoke mutator methods.

We next illustrate translation of a mutator method, using the following example from AbstractSet:

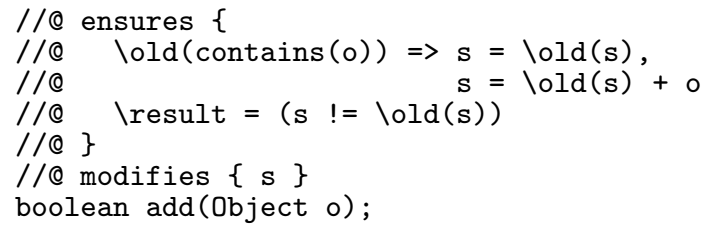

Again, the translation uses the postcondition to model the method, but it additionally uses the modifies annotation, because the method is a mutator. For a mutator method, the translation can also use the does annotation, which can include modifies.

The translation generates the following Alloy function:

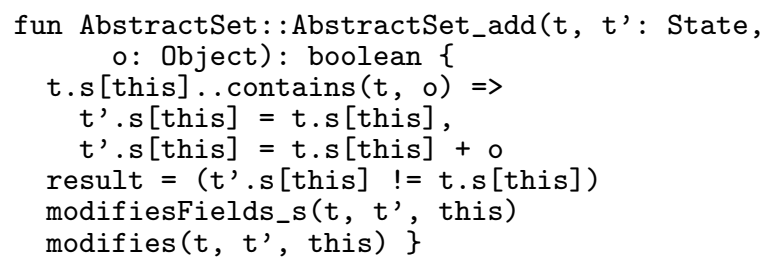

This function has, in addition to the method parameter, two State parameters, for pre-state $t$ and post-state $t$ '. We use primed notation to signify post-state; the prime mark has no built-in semantic significance in Alloy. The expressions prefixed with $\backslash$ old are translated to evaluate in the pre-state. The field accesses and the pure method invocation in the body of this general method are translated as illustrated earlier.

The modifies annotation, which specifies the modified fields, is translated into several function applications. For each set of fields that is modified, in this example $s$, an appropriate modifiesfields function is invoked with the set of objects, in this example this, that have exactly those fields modified, i.e., modifiesFields_s ( $t, t^{\prime}$ ', this). The frame condition modifiesFields_f constrains all the fields of an object but $f$ to be unchanged. If several fields are listed in the modifies clause, a composite frame condition must be generated, in the same way that a single modifies condition is generated for the whole set of objects that may change.

The function modifies is invoked with the set of objects that may be modified by the mutator method. This function specifies that the fields of all other objects remain unchanged:

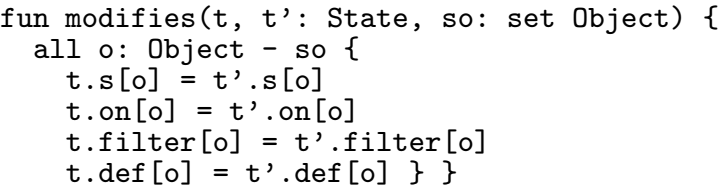

The function modifiesFields_s is similar, but it allows s to change:

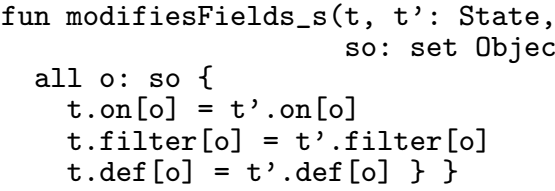

In general, the translation adds state(s) to an expression that appears in a specification depending on the expression (an invocation of a pure method pm, an invocation of a mutator method mm, or a field access) and whether the specification is for a pure method $P M$ (i.e., it has only one state) or for a mutator method $M M$ (i.e., it has a pre-state and a post-state):

- Invocation o..pm(...) in $P M$ or $\backslash$ old $(\circ \ldots$ pm $(\ldots))$ in $M M$ becomes o..pm(t, ...), where $\mathrm{t}$ is the (pre)state.

- Invocation o..pm(...) (not inside $\backslash$ old) in $M M$ becomes o..pm $(t, \ldots)$, where $t$ ' is the post-state.

- Invocation o..mm(..) in $M M$ becomes o..mm(t, t', ...).

- Field access o.f in $P M$ or $\backslash$ old(o.f) in $M M$ becomes $t . f[o]$.

- Field access o.f (not inside \old) in $M M$ becomes $t$ '. $f[0]$.

\subsection{Recursion}

The Alloy Analyzer currently does not support recursive functions. We allow AAL specifications to be recursive. Also, the translation given above results in recursive functions (in particular through dispatching functions). We next outline the translation that eliminates recursive functions so that the models can be analyzed with the current Alloy Analyzer.

Elimination of recursion introduces new relations in the signature State and constrains these relations using the corresponding functions. For each set of mutually recursive functions, the translation introduces a new relation; in our running example:

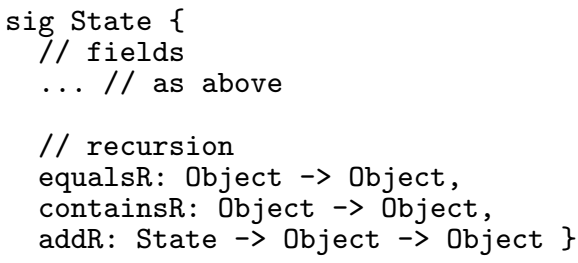


Since add is a mutator method, the type of its corresponding relation has an additional (post-)state.

For example, the translation eliminates recursion from equals by replacing it with:

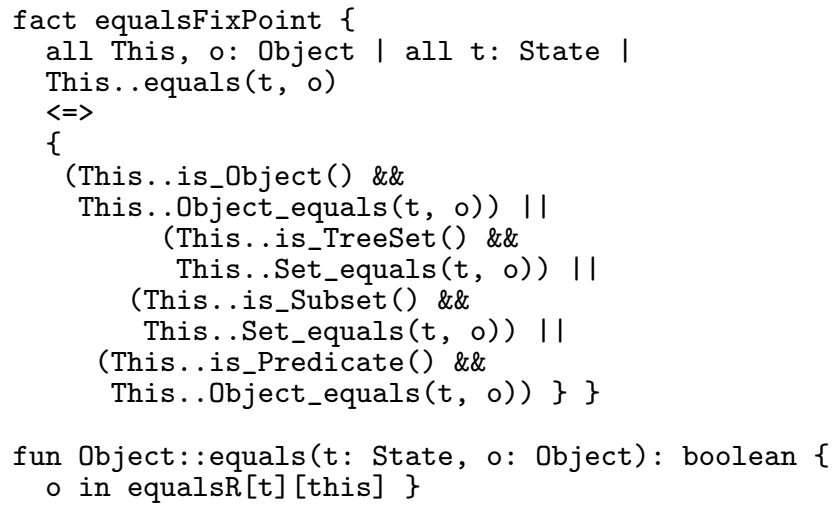

The fact equalsFixPoint defines the relation equalsR so that its tuples represent a fixed point of the equals methods. That is, it will contain a tuple $(s, 01,02)$ iff in state $s$, object $o 1$ is equal to object $\circ 2$; ' $\Leftrightarrow>$ ' denotes equivalence. Note that this translation does not constrain the relation to be the least fixed point; if the definitions of recursive functions have several fixed points, AA will explore all of them. This is an appropriate approach for relational specifications: a recursive definition with several fixed points corresponds simply to an underspecified behavior and the analysis should explore all possible behaviors.

The translation eliminates recursion from mutator methods in a similar fashion; for the add method:

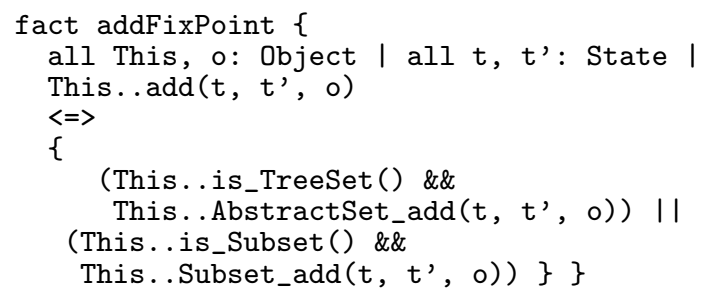

fun Object: :add( $t, t^{\prime}:$ State, o: Object): boolean \{ 0 in $\operatorname{addR}[t][t '][$ this $]\}$

\subsection{Object creation}

AAL supports object creation in mutator methods. The translation handles it by adding a field free: set object to the State signature; for each state $t$, $t$.free is the set of unallocated objects in that state. Object creation then allocates an object by removing it from the freeset.

The translation disallows the objects in $t$.free to be pointed to by any field. The translation also makes free objects to point to no object. For our running example, the translation adds:

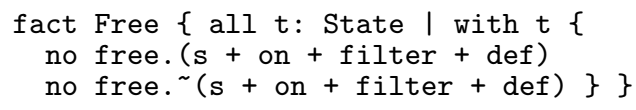

It also adds the following constraint to the body of the function modifies:

$t^{\prime}$.free $=t$.free - so

Consider the following Subset constructor for our running example:

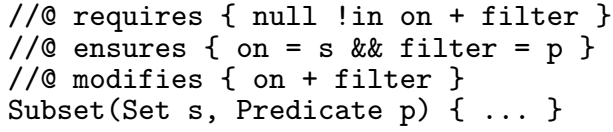

This constructor is translated to:

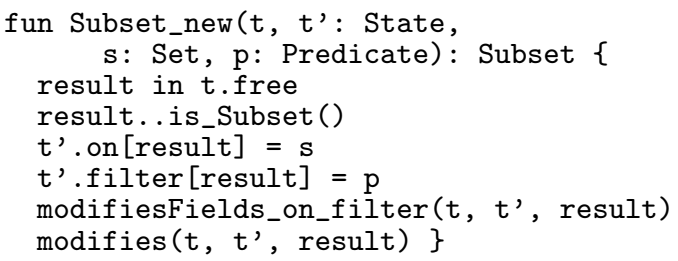

The function Subset_new requires result, i.e., the freshly allocated object, to be unallocated in the pre-state and also appropriately constrains its class. Subset_new also contains the translated modifies clause.

\subsection{Code sequences}

AAL supports analysis of (loop-free) code sequences that may have method invocations. Code with loops can be translated into loop-free code using, for example, a finite unrolling of loops $[4,15]$.

Consider, for example, the following code sequence that involves a conditional branch:

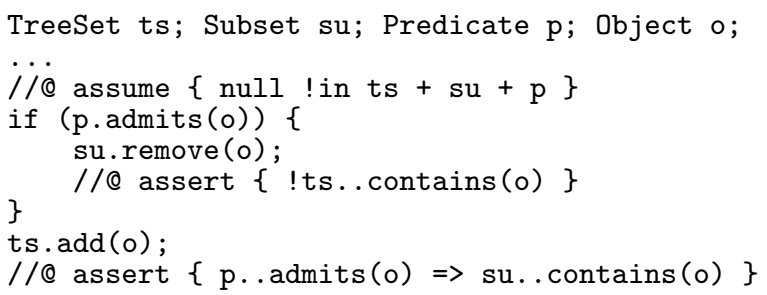

The translation uses verification condition generation, similar to that of ESC [4], and generates an Alloy assertion that threads state through the sequence as shown in Figure 2. This translation requires all (post-)states to be valid. If methods are already checked to be correct implementations, then this requirement can be omitted.

Note that all local variables declared in this example do not change their values in the sequence and thus can be universally quantified. In general, a local variable can be bound by several assignment statements in a code sequence, so it cannot be translated simply to a quantified variable. One can handle this by treating local variables as "fields" of the state. Alternatively, instances of the same local variable can be given distinct names, putting the code into single static assignment (SSA) form [2]. This tends to result in smaller Alloy formulas and thus faster analysis. 


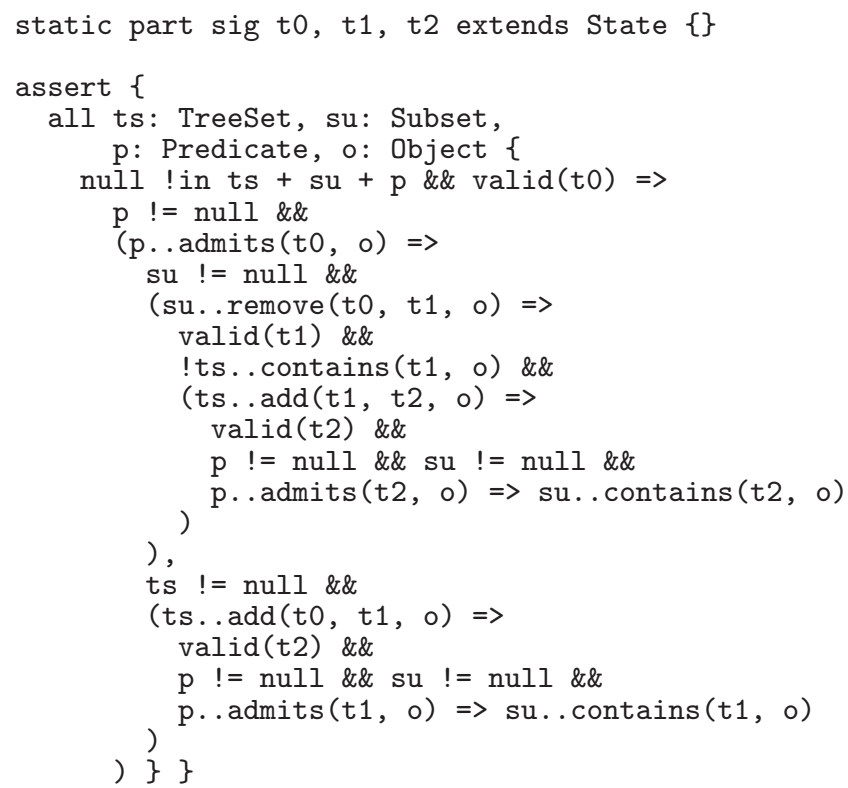

Figure 2: Translation of the example code sequence.

\section{Related work}

Recent annotation languages, such as the Java Modeling Language (JML) [19] and the assertion subset of Eiffel [26], are themselves based on much earlier foundational work in program annotation. ANNA [22], for example, was an early annotation language for Ada; the Larch interface languages, such as Larch/C++ [18] led the way for modeling of the heap in an object-oriented language. Eiffel's innovation was to integrate pre- and post-conditions into the programming language itself, and to apply the assertions of superclasses automatically to their subclasses. A systematic analysis of the relationship between the assertions of classes at different levels in the class hierarchy has been developed recently by Findler and Felleisen [6]. Eiffel typifies the languages, like JML, that build on the expression language of the programming language; ANNA and Larch/C++ typify those languages, like AAL, that build assertions on a more abstract semantics.

Our work builds on these foundations. Its novelty is twofold: using a relational semantics of the heap, and providing opportunities for automatic compile-time analysis based on exhaustive search. These aspects are intertwined: our analysis technology relies on the uniform representation of data structures as relations, which are easily encoded as boolean matrices, making possible the application of SAT solving technology. The use of relations as a modeling tool for data structures is not new; it goes back to the earliest work on data abstraction [5], and to the entire field of semantic data modeling. Specification and assertion languages for objectoriented programs have, however, invariably preferred algebraic semantics instead, despite the use of graphs in shape analysis as abstractions of the heap.

To our knowledge, our work is unique in offering the prospect of fully automatic compile-time analysis for annotations that can capture complex structural properties. Several other annotation languages that are equally rich (or richer) have been developed, such as JML and the assertion subset of Eiffel, but these have been designed primarily for run-time checking.

Static analyzers, such as the Extended Static Checker [4], the Three-Valued-Logic Analyzer [21,31], the Pointer Assertion Logic Engine [27], and Role Analysis [17], are automatic, but cannot handle such expressive annotations. None of these, to our knowledge, for example, can express and analyze the red-black invariants (at least without additional manual instrumentation).

Our analysis would be termed unsound in the program analysis literature: that is, it may fail to find a counterexample if one exists. Since we view its purpose as the discovery of flaws rather than the demonstration of correctness, we prefer to think of it as incomplete: not all bugs are found, but it is sound in the sense that any counterexample reported will represent a legitimate execution. None of the analyses mentioned above reliably produce sound counterexamples. In fact, most are also not complete: the authors of ESC have argued convincingly that making their checker complete would cause it to generate many more false alarms.

The LOOP project [35] aims at proving properties of Java classes by translating them and their JML specifications into logical theories in higher order logic. A theorem prover is then used to verify the desired properties. This framework has been used to verify that the methods of java.util.Vector maintain the safety property that the actual size of a vector is less than or equal to its capacity [8].

Our analysis translates AAL annotations and Java code into Alloy. Alloy has been previously used for object-oriented modeling. Two previous works propose encodings of the heap in Alloy: one, like ours, models references directly as relations [12]; the other [13] represents mutations as changes in the mapping of references to mathematical objects. This second approach is more modular, but results in a less tractable analysis. AAL builds on these approaches by adding direct support for (single) dynamic dispatch and recursion, and by setting these constructs in the context of an annotation language.

The approach to checking code against partial specifications that we advocate in this paper is described in more detail elsewhere [15] and is the subject of ongoing work [36]. Although it has yet to be shown to scale, it has been applied successfully to a practical implementation of a red-black tree.

Marinov and Khurshid developed TestEra [24], an Alloybased framework for automated testing of Java programs. In TestEra, specifications are written in Alloy, and the Alloy Analyzer is used both as an automatic test case generator and as a test oracle. TestEra discovered bugs in a previous version of the Alloy Analyzer. TestEra has also been used to systematically test data structures, such as red-black trees.

Formal specification languages have been also extended with object-oriented features for greater expressiveness and for 
more direct modeling of object-oriented systems. Object$\mathrm{Z}$ [32], for example, extends the $\mathrm{Z}$ specification language [33] with a new kind of schema in which operations may be associated with instances of a class. Object- $Z$ is not used as an annotation language, and is not amenable to automatic analysis.

Objects and inheritance have also been added to declarative languages. For example, Prolog ++ [28] extends Prolog. $\mathrm{OOLP}+[3]$ aims to integrate object-oriented paradigm with logic programming by translating OOLP + code into Prolog without meta-interpretation.

Keidar et al. [16] add inheritance to the IOA language [23] for modeling state machines, which enables reusing simulation proofs between state machines. This approach allows only a limited form of inheritance, subclassing for extension: subclasses can add new methods and specialize inherited methods, but they cannot override those inherited methods, changing their behavior arbitrarily. AAL allows subclasses that arbitrarily change the behavior of inherited methods, as illustrated with the equals methods.

\section{Conclusions}

The key idea in this paper is that a simple logic with relational operators suffices as a semantic basis for an annotation language. The choice of this logic was motivated by the desire for fully automatic analysis of object-oriented programs. The logic, due to its relational operators, has a direct appeal from a specification viewpoint also. Navigation expressions (especially those involving transitive closure) are succinct and easy to write; witness the red-black tree invariants of Section 3 .

The analysis upon which our scheme relies has two properties that make it well suited to analysis of object-oriented programs. First, a huge space of cases can be covered; the analysis therefore tends to find subtle problems much more readily than testing. Second, when a claim does not hold, the analysis produces a counterexample: one or more configurations of the heap that show why the claim is invalid.

Our examples have shown that this approach can be applied to program structures that are known to be a cause of complexity and errors. Currently, our translations are done systematically but manually; the analysis is performed fully automatically by the Alloy Analyzer (and counterexamples are displayed using its visualization facility). We are now implementing the translation outlined in this paper in order to build a comprehensive tool. We expect that the tool will work well for modular analysis of even quite complex classes; how well it scales for analyses amongst classes, and whether it will be economical enough for everyday use, remains to be seen.

\section{Acknowledgments}

We would like to thank Matthias Felleisen, Viktor Kuncak, and Gregory Sullivan for discussions on AAL and comments on an earlier draft of this paper. This work was funded in part by ITR grant \#0086154 from the National Science Foundation.

\section{References}

[1] K. Beck. Extreme Programming Explained: Embrace Change. Addison-Wesley, 2000.

[2] D. R. Chase, M. Wegman, and F. K. Zadeck. Analysis of pointers and structures. In Proc. ACM SIGPLAN'90 Conference on Programming Language Design and Implementation (PLDI), pages 296-310, White Plains, N.Y., June 1990.

[3] M. Dalal and D. Gangopahyay. OOLP: A translation approach to object-oriented logic programming. In Proc. First International Conference on Deductive and Object-Oriented Databases (DOOD-89), pages 555-568, Kyoto, Japan, Dec. 1989.

[4] D. L. Detlefs, K. R. M. Leino, G. Nelson, and J. B. Saxe. Extended static checking. Research Report 159, Compaq Systems Research Center, 1998.

[5] J. Earley. Toward an understanding of data structures. Communications of the ACM, 14(10):617-627, 1971.

[6] R. Findler and M. Felleisen. Contract soundness for object-oriented languages. In Proc. ACM SIGPLAN 2001 Conference on Object-Oriented Programming, Systems, Languages, and Applications (OOPSLA), pages 1-15, Oct. 2001.

[7] R. Helm, I. M. Holland, and D. Gangopadhyay. Contracts: Specifying behavioral compositions in object-oriented systems. In Proc. of the OOPSLA/ECOOP-90: Conference on Object-Oriented Programming Systems, Languages, and Applications, pages 169-180, Ottawa, Canada, 1990.

[8] M. Huisman, B. Jacobs, and J. van den Berg. A case study in class library verification: Java's Vector class. Software Tools for Technology Transfer, 2001.

[9] D. Jackson. Automating first-order relational logic. In Proc. 8th ACM SIGSOFT Symposium on the Foundations of Software Engineering (FSE), San Diego, CA, November 2000.

[10] D. Jackson. Micromodels of software: Modelling and analysis with Alloy, 2001. Available online: http://sdg.lcs.mit.edu/alloy/book.pdf.

[11] D. Jackson. Alloy: A lightweight object modeling notation. ACM Transactions on Software Engineering and Methodology (TOSEM), 11(2), Apr. 2002.

[12] D. Jackson. Object models as heap invariants. In C. Morgan and A. McIver, editors, Essays on Programming Methodology. Springer Verlag, 2002. (to appear).

[13] D. Jackson and A. Fekete. Lightweight analysis of object interactions. In Proc. Fourth International Symposium on Theoretical Aspects of Computer Software, Sendai, Japan, Oct. 2001.

[14] D. Jackson, I. Shlyakhter, and M. Sridharan. A micromodularity mechanism. In Proc. 9th ACM SIGSOFT Symposium on the Foundations of Software Engineering (FSE), Vienna, Austria, Sept. 2001. 
[15] D. Jackson and M. Vaziri. Finding bugs with a constraint solver. In Proc. International Symposium on Software Testing and Analysis (ISSTA), Portland, OR, Aug. 2000.

[16] I. Keidar, R. Khazan, N. Lynch, and A. Shvartsman. An inheritance-based technique for building simulation proofs incrementally. In Proc. 22nd International Conference on Software Engineering (ICSE), pages 478-487, Limerick, Ireland, June 2000.

[17] V. Kuncak, P. Lam, and M. Rinard. Role analysis. In Proc. 29th Annual ACM Symposium on the Principles of Programming Languages (POPL), Portland, OR, Jan. 2002.

[18] G. T. Leavens. An overview of Larch/C++: Behavioral specifications for $\mathrm{C}++$ modules. In H. Kilov and W. Harvey, editors, Specification of Behavioral Semantics in Object-Oriented Information Modeling, pages 121-142. Kluwer Academic Publishers, 1996.

[19] G. T. Leavens, A. L. Baker, and C. Ruby. Preliminary design of JML: A behavioral interface specification language for Java. Technical Report TR 98-06i, Department of Computer Science, Iowa State University, June 1998. (last revision: Aug 2001).

[20] K. R. M. Leino. Data groups: Specifying the modification of extended state. In Proc. ACM SIGPLAN 1998 Conference on Object-Oriented Programming, Systems, Languages, and Applications (OOPSLA), volume 33, pages 144-153, Oct. 1998.

[21] T. Lev-Ami and M. Sagiv. TVLA: A system for implementing static analyses. In Proc. Static Analysis Symposium, Santa Barbara, CA, June 2000.

[22] D. C. Luckham and F. von Henke. An overview of Anna, a specification language for Ada. In IEEE Software, volume 2, pages 9-23, Mar. 1985.

[23] N. Lynch. Distributed Algorithms. Morgan Kaufmann Publishers, 1996.

[24] D. Marinov and S. Khurshid. TestEra: A novel framework for automated testing of Java programs. In Proc. 16th IEEE International Conference on Automated Software Engineering (ASE), San Diego, CA, Nov. 2001.

[25] D. Marinov and S. Khurshid. VAlloy: Virtual functions meet a relational language. In Proc. Formal Methods Europe (FME), Copenhagen, Denmark, July 2002.

[26] B. Meyer. Eiffel: The Language. Prentice Hall, New York, N.Y., 1992.

[27] A. Moeller and M. I. Schwartzbach. The pointer assertion logic engine. In Proc. SIGPLAN Conference on Programming Languages Design and Implementation, Snowbird, UT, June 2001.

[28] C. Moss. Prolog ++ The Power of Object-Oriented and Logic Programming. Addison-Wesley, 1994.
[29] P. Müller, A. Poetzsch-Heffter, and G. T. Leavens. Modular specification of frame properties in JML. Technical Report 02-02, Iowa State University, Feb. 2002.

[30] M. Roulo. How to avoid traps and correctly override methods from java.lang. Object. http://www . javaworld.com/javaworld/jw-01-1999/ jw-01-object.html.

[31] M. Sagiv, T. Reps, and R. Wilhelm. Solving shape-analysis problems in languages with destructive updating. ACM Transactions on Programming Languages and Systems (TOPLAS), January 1998.

[32] G. Smith. The Object-Z Specification Language. Kluwer Academic Publishers, 2000.

[33] J. M. Spivey. The Z Notation: A Reference Manual. Prentice Hall, second edition, 1992.

[34] Sun Microsystems. Java 2 Platform, Standard Edition, v1.3.1 API Specification. http://java.sun.com/j2se/1.3/docs/api/.

[35] J. van den Berg and B. Jacobs. The LOOP compiler for Java and JML. In Proc. Tools and Algorithms for the Construction and Analysis of Software (TACAS), (Springer LNCS 2031, 2001), pages 299-312, Genoa, Italy, Apr. 2001.

[36] M. Vaziri. Finding bugs with a constraint solver. PhD Thesis Proposal, Dept. of Electrical Engineering and Computer Science, Massachusetts Institute of Technology, Cambridge, MA, 2002. 